\title{
On general reduced second Zagreb index of graphs
}

\author{
Batmend Horoldagva ${ }^{1,2}$ (D), Lkhagva Buyantogtokh ${ }^{3}$ (D), Kinkar Ch. Das *3 (D), \\ Sang-Gu Lee ${ }^{3}$ (D) \\ ${ }^{1}$ Department of Mathematics, Mongolian National University of Education, Baga toiruu-14, Ulaanbaatar, \\ Mongolia \\ ${ }^{2}$ Institute of Mathematics, National University of Mongolia, Baga toiruu-1, Ulaanbaatar, Mongolia \\ ${ }^{3}$ Department of Mathematics, Sungkyunkwan University, Suwon 440-746, Republic of Korea
}

\begin{abstract}
Recently, Furtula et al. [B. Furtula, I. Gutman, S. Ediz, On difference of Zagreb indices, Discrete Appl. Math., 2014] introduced a new vertex-degree-based graph invariant "reduced second Zagreb index" in chemical graph theory. Here we generalize the reduced second Zagreb index (call "general reduced second Zagreb index"), denoted by $G R M_{\alpha}(G)$ and is defined as: $G R M_{\alpha}(G)=\sum_{u v \in E(G)}\left(d_{G}(u)+\alpha\right)\left(d_{G}(v)+\alpha\right)$, where $\alpha$ is any real number and $d_{G}(v)$ is the degree of the vertex $v$ of $G$. Let $\mathcal{G}_{n}^{k}$ be the set of connected graphs of order $n$ with $k$ cut edges. In this paper, we study some properties of $G R M_{\alpha}(G)$ for connected graphs $G$. Moreover, we obtain the sharp upper bounds on $G R M_{\alpha}(G)$ in $\mathcal{G}_{n}^{k}$ for $\alpha \geq-1 / 2$ and characterize the extremal graphs.
\end{abstract}

Mathematics Subject Classification (2010). 05C07, 05C12, 05C30

Keywords. Connected graph, Zagreb indices, general reduced second Zagreb index, cut edge, maximum degree

\section{Introduction}

Let $G=(V, E)$ be a connected graph with vertex set $V(G)$ and edge set $E(G)$. Denote by $d_{G}(u)$, the degree of the vertex $u$ of $G$. A graph invariant is a number related to a graph which is a structural invariant, in other words, it is a fixed number under graph automorphisms. The oldest and well-known graph invariants are the classical Zagreb indices $\left(M_{1}\right.$ and $\left.M_{2}\right)$ of graph $G$ and they are defined as

$$
M_{1}(G)=\sum_{u \in V(G)}\left(d_{G}(u)\right)^{2} \quad \text { and } \quad M_{2}(G)=\sum_{u v \in E(G)} d_{G}(u) d_{G}(v) .
$$

The Zagreb indices $M_{1}$ and $M_{2}$ were first introduced by Gutman and Trinajstić in 1972, the quantities of the Zagreb indices were found to occur within certain approximate expressions for the total $\pi$-electron energy [15]. In 1975, these graph invariants were proposed to be measures of branching of the carbon atom skeleton [14]. For details of the mathematical theory and chemical applications of the Zagreb indices, see [2, 7, 9, 13,22]. The Zagreb

\footnotetext{
*Corresponding Author.

Email addresses: horoldagva@msue.edu.mn (B. Horoldagva), buyantogtokh.lhag@yahoo.com (L. Buyantogtokh), kinkardas2003@gmail.com (K.Ch. Das), sglee@skku.edu ( S.-G. Lee)

Received: 10.8.2017; Accepted: 06.02.2018
} 
indices were independently studied in the mathematical literature under other names $[1,6,21,27]$.

Caporossi and Hansen [3] conjectured that, for all connected graphs $G$ it holds that

$$
\frac{M_{1}(G)}{n} \leq \frac{M_{2}(G)}{m}
$$

and the bound is tight for complete graphs. Although this conjecture is disproved for general graphs [16], it was the beginning of a long series of studies in which the validity or non-validity of inequality (1.1) for various classes of graphs, see [16,18, 20,25, 26] and the references cited therein.

Recently, much attention is being paid to the comparison of $M_{1}$ and $M_{2}$. Direct comparisons were obtained on the Zagreb indices for trees [8,24] and cyclic graphs [4]. The difference of the Zagreb indices of a graph $G$ has been studied in $[12,19]$.

Furtula, Gutman and Ediz [12] showed that the difference of the Zagreb indices is closely related to the vertex-degree-based graph invariant

$$
R M_{2}(G)=\sum_{u v \in E(G)}\left(d_{G}(u)-1\right)\left(d_{G}(v)-1\right)
$$

and determined a few basic properties of $M R_{2}$. This vertex-degree-based graph invariant $R M_{2}$ is called reduced second Zagreb index and it was studied in [12] for trees and in [17] for cyclic graphs with cut edges.

Here we generalize the reduced second Zagreb index (call "general reduced second Zagreb index"), denoted by $G R M_{\alpha}(G)$ and is defined as:

$$
G R M_{\alpha}(G)=\sum_{u v \in E(G)}\left(d_{G}(u)+\alpha\right)\left(d_{G}(v)+\alpha\right),
$$

where $\alpha$ is any real number. It was studied in [1] for general graphs when $\alpha=1$.

A pendant vertex is a vertex of degree one. An edge of a graph is said to be pendant if one of its end vertices is a pendant vertex. For $v \in V(G), N_{G}(v)$ denotes the neighbors of $v$ and $N_{G}[v]=N_{G}(v) \cup\{v\}$. The maximum vertex degree of $G$ is denoted by $\Delta(G)$. Denote by $\bar{G}$, the complement of graph $G$. A cut edge in a graph $G$ is an edge whose removal increases the number of connected components of $G$. For a subset $E^{\prime}$ of $E(G)$, we denote by $G-E^{\prime}$ the subgraph of $G$ obtained by deleting the edges in $E^{\prime}$. For a subset $E^{\prime \prime}$ of $E(\bar{G})$, the graph obtained from $G$ by adding a set of edges $E^{\prime \prime}$ is denoted by $G+E^{\prime \prime}$. If $E^{\prime}=\left\{e_{1}\right\}$ and $E^{\prime \prime}=\left\{e_{2}\right\}$, the subgraph $G-E^{\prime}$ and the super graph $G+E^{\prime \prime}$ will be written as $G-e_{1}$ and $G+e_{2}$ for short, respectively. Denote by $\mathcal{G}_{n}^{k}$ the set of connected graphs of order $n$ with $k$ cut edges. Let $K_{n}^{k}$ be a graph obtained by joining $k$ pendant vertices to one vertex of the complete graph $K_{n-k}$. Also denote by $\mathcal{G}_{n}^{k+}$ the set of connected graphs of order $n$ with at least $k$ cut edges. Then we have $K_{n}^{k} \in \mathcal{G}_{n}^{k}, \mathcal{G}_{n}^{k} \subseteq \mathcal{G}_{n}^{k+}$ and $\mathcal{G}_{n}^{k+}=\bigcup_{i \geq k} \mathcal{G}_{n}^{i}$. Note that a connected graph of order $n$ has at most $n-1$ cut edges.

The extremal graphs of order $n$ with $k$ cut edges on Zagreb indices were studied in $[10,11]$. Namely, it was proved that $K_{n}^{k}$ has maximum $M_{1}$ or $M_{2}$-value in $\mathcal{G}_{n}^{k}$. Alternative proof of these results were given in [5].

This paper is organized as follows. In Section 2, some properties of $G R M_{\alpha}$ are provided. In Section 3, we present the sharp upper bound on $G R M_{\alpha}$ in $\mathcal{G}_{n}^{k}$ for $\alpha>-1 / 2$ and characterize the extremal graphs. In Section 4, we obtain the sharp upper bound on $G R M_{\alpha}$ in $\mathcal{G}_{n}^{k}$ for $\alpha=-1 / 2$ and characterize the extremal graphs. 


\section{Properties of $G R M_{\alpha}$}

In this section, we provide some properties of $G R M_{\alpha}$ that will be useful in our study in later sections. From the definitions of $M_{1}$ and $M_{2}$, we easily get the following identity

$$
\begin{aligned}
G R M_{\alpha}(G) & =\sum_{u v \in E(G)}\left(d_{G}(u)+\alpha\right)\left(d_{G}(v)+\alpha\right) \\
& =\sum_{u v \in E(G)} d_{G}(u) d_{G}(v)+\alpha \sum_{u v \in E(G)}\left(d_{G}(u)+d_{G}(v)\right)+\alpha^{2}|E(G)| \\
& =M_{2}(G)+\alpha M_{1}(G)+\alpha^{2}|E(G)|
\end{aligned}
$$

where $\alpha$ is any real number.

Lemma 2.1. Let $G$ be a connected graph. Let $u v \in E(G)$ and $N_{G}(v) \backslash N_{G}[u]=\left\{v_{1}, v_{2}, \ldots, v_{t}\right\} \neq$ $\emptyset$. Consider the graph $G^{\prime}=G-\left\{v v_{1}, v v_{2}, \ldots, v v_{t}\right\}+\left\{u v_{1}, u v_{2}, \ldots, u v_{t}\right\}$. Then

(i) $M_{1}\left(G^{\prime}\right)-M_{1}(G)=2 t\left(\left(d_{G}(u)-d_{G}(v)+t\right)\right.$,

(ii) $M_{2}\left(G^{\prime}\right)-M_{2}(G) \geq t\left(d_{G}(u)-d_{G}(v)+t\right)$ when $d_{G}(u) \geq d_{G}(v)$.

Proof. Now we have $d_{G}(w)=d_{G^{\prime}}(w)$ for $w \neq u, v$ whereas $d_{G^{\prime}}(u)=d_{G}(u)+t$ and $d_{G^{\prime}}(v)=d_{G}(v)-t$.

(i) By the definition of $M_{1}$, we get

$$
\begin{aligned}
M_{1}\left(G^{\prime}\right)-M_{1}(G) & =\left(d_{G}(u)+t\right)^{2}+\left(d_{G}(v)-t\right)^{2}-d_{G}(u)^{2}-d_{G}(v)^{2} \\
& =2 t\left(\left(d_{G}(u)-d_{G}(v)+t\right) .\right.
\end{aligned}
$$

(ii) Also, by the definition of $M_{2}$, we get

$$
\begin{gathered}
M_{2}\left(G^{\prime}\right)-M_{2}(G)=\sum_{x \in N_{G}(u) \backslash N_{G}[v]}\left(d_{G}(u)+t\right) d_{G}(x)+\sum_{i=1}^{t}\left(d_{G}(u)+t\right) d_{G}\left(v_{i}\right) \\
+\sum_{y \in N_{G}(u) \cap N_{G}(v)}\left(d_{G}(u)+d_{G}(v)\right) d_{G}(y)+\left(d_{G}(u)+t\right)\left(d_{G}(v)-t\right) \\
\quad-\sum_{x \in N_{G}(u) \backslash N_{G}[v]} d_{G}(u) d_{G}(x)-\sum_{i=1}^{t} d_{G}(v) d_{G}\left(v_{i}\right) \\
\quad-\sum_{y \in N_{G}(u) \cap N_{G}(v)}\left(d_{G}(u)+d_{G}(v)\right) d_{G}(y)-d_{G}(u) d_{G}(v) \\
=\sum_{x \in N_{G}(u) \backslash N_{G}[v]} t d_{G}(x)+\sum_{i=1}^{t}\left(d_{G}(u)-d_{G}(v)+t\right) d_{G}\left(v_{i}\right) \\
\\
=\sum_{x \in N_{G}(u) \backslash N_{G}[v]} t d_{G}(x)+\sum_{i=1}^{t}\left(d_{G}(u)-d_{G}(v)+t\right)\left(d_{G}\left(v_{i}\right)-1\right) \\
\geq \sum_{x \in N_{G}(u) \backslash N_{G}[v]} t d_{G}(x) \geq t\left|N_{G}(u) \backslash N_{G}[v]\right|
\end{gathered}
$$


since $G$ is connected and $d_{G}(u) \geq d_{G}(v)$.

It is easy to see that $N_{G}(u) \cup\left(N_{G}(v) \backslash N_{G}[u]\right)=N_{G}(v) \cup\left(N_{G}(u) \backslash N_{G}[v]\right)$. Therefore we have

$$
\left|N_{G}(u)\right|+\left|N_{G}(v) \backslash N_{G}[u]\right|=\left|N_{G}(v)\right|+\left|N_{G}(u) \backslash N_{G}[v]\right|,
$$

that is

$$
d_{G}(u)+t=d_{G}(v)+\left|N_{G}(u) \backslash N_{G}[v]\right| .
$$

From (2.2) and (2.3), we get the required result. This completes the proof.

Lemma 2.2. Let $G$ be a connected graph. Let $u v \in E(G)$ and $N_{G}(v) \backslash N_{G}[u]=\left\{v_{1}, v_{2}, \ldots, v_{t}\right\}$. Consider the graph $G^{\prime}=G-\left\{v v_{1}, v v_{2}, \ldots, v v_{t}\right\}+\left\{u v_{1}, u v_{2}, \ldots, u v_{t}\right\}$. Then the number of cut edges in $G$ is less than or equal to the number of cut edges in $G^{\prime}$.

Proof. We prove that the number of non-cut edges in $G^{\prime}$ is less than or equal to the number of non-cut edges in $G$. Obviously, $\left|E\left(G^{\prime}\right)\right|=|E(G)|$. Hence it is sufficient to prove that for every non-cut edge in $G^{\prime}$, there is a corresponding non-cut edge in $G$.

If $u v$ is a non-cut edge in $G^{\prime}$, then it is also non-cut edge in $G$. Conversely, suppose that $u v$ is a cut edge in $G$. Then $N_{G}(u) \cap N_{G}(v)=\emptyset$ and it follows that $u v$ is a pendant edge in $G^{\prime}$. But this contradicts the fact that $u v$ is a non-cut edge in $G^{\prime}$.

For $1 \leq i \leq t$, if $u v_{i}$ is a non-cut edge in $G^{\prime}$, then there is a path $Q\left(Q \neq u v_{i}\right)$ from $u$ to $v_{i}$ in $G^{\prime}$. Obviously $Q$ is the subgraph of $G^{\prime}$. For the convenience, we denote by $E(Q)$ the edge set of $Q$. If $u v \in E(Q)$ then $Q-u v$ is a path from $v$ to $v_{i}$ in $G$. Otherwise $Q+u v$ is a path from $v$ to $v_{i}$ in $G$. Therefore $v v_{i}$ is a non-cut edge in $G$ for $1 \leq i \leq t$.

Now the proof will be completed by showing that if $x y$ is a non-cut edge in $G^{\prime}$, which is different from $u v_{1}, u v_{2}, \ldots, u v_{t}$ and $u v$, then $x y$ must also be a non-cut edge in $G$. Since $x y$ is a non-cut edge in $G^{\prime}$, there is a path $P(P \neq x y)$ from $x$ to $y$ in $G^{\prime}$. Since $P$ is the path, there are at most two edges incident to $u$ in $E(P)$. If $u v_{i} \notin E(P)$ for each $1 \leq i \leq t$, then $P$ is a path from $x$ to $y$ in $G$. Thus $x y$ is a non-cut edge in $G$. Let now $u v_{i} \in E(P)$ and $u v_{s} \notin E(P)$ for each $1 \leq s \leq t$ such that $s \neq i$. In this case, if $u v \in E(P)$ then $P-\left\{u v, u v_{i}\right\}+v v_{i}$ is a path from $x$ to $y$ in $G$. Otherwise $P-u v_{i}+\left\{u v, v v_{i}\right\}$ is a path from $x$ to $y$ in $G$. Finally if $u v_{i} \in E(P), u v_{j} \in E(P)$ and $u v_{s} \notin E(P)$ for each $1 \leq s \leq t$ such that $s \neq i, j$, then $P-\left\{u v_{i}, u v_{j}\right\}+\left\{v v_{i}, v v_{j}\right\}$ is a path from $x$ to $y$ in $G$. This completes the proof.

Proposition 2.3. Let $G$ be a graph in $\mathcal{G}_{n}^{k+}$ and $\alpha>-1 / 2$. If $G R M_{\alpha}(G)$ is maximum, then we have $\Delta(G)=n-1$.

Proof. By contradiction we will prove this result. For this let $u$ be a maximum degree vertex in $G$ and $d(u)<n-1$. Since $G$ is connected, there exist the vertices $v$ and $v_{1}$ in $G$ such that $u v, v v_{1} \in E(G)$ and $u v_{1} \notin E(G)$, where $v_{1}$ is the vertex at distance 2 from $u$. Obviously, $v_{1} \in N_{G}(v) \backslash N_{G}[u]$ and let $N_{G}(v) \backslash N_{G}[u]=\left\{v_{1}, v_{2}, \ldots, v_{t}\right\}$. Now we consider the graph

$$
G^{\prime}=G-\left\{v v_{1}, v v_{2}, \ldots, v v_{t}\right\}+\left\{u v_{1}, u v_{2}, \ldots, u v_{t}\right\} .
$$

Then, we have $G^{\prime} \in \mathcal{G}_{n}^{k+}$ by Lemma 2.2. Obviously, $\left|E\left(G^{\prime}\right)\right|=|E(G)|$.

Hence by Lemma 2.1 , we get

$$
\begin{aligned}
G R M_{\alpha}\left(G^{\prime}\right)-G R M_{\alpha}(G) & =M_{2}\left(G^{\prime}\right)-M_{2}(G)+\alpha\left(M_{1}\left(G^{\prime}\right)-M_{1}(G)\right) \\
& \geq t(1+2 \alpha)\left(d_{G}(u)-d_{G}(v)+t\right)>0
\end{aligned}
$$


since $u$ is the maximum degree vertex, $t \geq 1$ and $\alpha>-1 / 2$. Therefore $G R M_{\alpha}\left(G^{\prime}\right)>$ $G R M_{\alpha}(G)$ for $\alpha>-1 / 2$, but it contradicts the fact that $G R M_{\alpha}(G)$ is maximum in $\mathcal{G}_{n}^{k+}$. Hence $\Delta(G)=n-1$.

Corollary 2.4. Let $T$ be a tree of order $n$ and $\alpha>-1 / 2$. If $G R M_{\alpha}(T)$ is maximum, then $T$ is isomorphic to star graph $S_{n}$.

Proposition 2.5. Let $G$ be a connected graph and $\alpha \geq-1$. Also let $u v \notin E(G)$. Consider the graph $G^{\prime}=G+u v$. Then

$$
G R M_{\alpha}\left(G^{\prime}\right)>G R M_{\alpha}(G)
$$

Proof. We have $d_{G}(w)=d_{G^{\prime}}(w)$ for $w \neq u, v$ whereas $d_{G^{\prime}}(u)=d_{G}(u)+1$ and $d_{G^{\prime}}(v)=$ $d_{G}(v)+1$. Hence by the definition of $G R M_{\alpha}$, we get

$$
\begin{aligned}
& \operatorname{GRM}_{\alpha}\left(G^{\prime}\right)-G R M_{\alpha}(G) \\
= & \sum_{x y \in E\left(G^{\prime}\right)}\left(d_{G^{\prime}}(x)+\alpha\right)\left(d_{G^{\prime}}(y)+\alpha\right)-\sum_{x y \in E(G)}\left(d_{G}(x)+\alpha\right)\left(d_{G}(y)+\alpha\right) \\
= & \sum_{x \in N_{G}(u)}\left(d_{G}(x)+\alpha\right)\left(d_{G}(u)+1+\alpha\right)+\sum_{x \in N_{G}(v)}\left(d_{G}(x)+\alpha\right)\left(d_{G}(v)+1+\alpha\right) \\
& +\left(d_{G}(u)+1+\alpha\right)\left(d_{G}(v)+1+\alpha\right) \\
& \quad-\sum_{x \in N_{G}(u)}\left(d_{G}(x)+\alpha\right)\left(d_{G}(u)+\alpha\right)-\sum_{x \in N_{G}(v)}\left(d_{G}(x)+\alpha\right)\left(d_{G}(v)+\alpha\right) \\
= & \sum_{x \in N_{G}(u)}\left(d_{G}(x)+\alpha\right)+\sum_{x \in N_{G}(v)}\left(d_{G}(x)+\alpha\right)+\left(d_{G}(u)+1+\alpha\right)\left(d_{G}(v)+1+\alpha\right) \\
> & 0
\end{aligned}
$$

since $d_{G}(z) \geq 1$ for all $z \in V(G)$ and $\alpha \geq-1$. This completes the proof.

An edge $u v$ of a graph $G$ is said to be contracted if it is deleted and its end vertices $u$ and $v$ are identified, the obtained graph is denoted by $G \cdot u v$. Also the identified vertex in $G \cdot u v$ is denoted by one of $u$ and $v$. A double-star is a tree with exactly two vertices of degree greater than 1. Obviously, a double-star has a unique non-pendant cut edge. Denote by $\mathcal{G}_{n, m}$, the set of connected graphs of order $n$ with $m$ edges.

Proposition 2.6. Let $G$ be a graph in $\mathcal{G}_{n, m}$. Also let $G R M_{\alpha}(G)$ be maximum.

(i) If $\alpha>-1 / 2$ then all cut edges of $G$ are pendant.

(ii) If $\alpha=-1 / 2$ and $G$ is different from a double-star, then all cut edges of $G$ are pendant.

Proof. We prove this result by contradiction. For this let $G$ be a graph with at least one non-pendant cut edge $u v$ in $\mathcal{G}_{n, m}$ and $\alpha \geq-1 / 2$ such that $G R M_{\alpha}(G)$ is maximum. Let $G^{\prime}$ be a graph obtained from $G \cdot u v$ by joining a pendant vertex $x$ to the identified vertex $u$. Then we have $G^{\prime} \in \mathcal{S}_{n, m}$. Also, we have $d_{G^{\prime}}(x)=1$ and $d_{G^{\prime}}(\omega)=d_{G}(\omega)$ for $\omega \neq u$ 
whereas $d_{G^{\prime}}(u)=d_{G}(u)+d_{G}(v)-1$. Therefore, we have

$$
\begin{aligned}
M_{2}\left(G^{\prime}\right)-M_{2}(G)= & \left(d_{G}(u)+d_{G}(v)-1\right)\left(1+\sum_{u_{i} \in N_{G}(u) \backslash\{v\}} d_{G}\left(u_{i}\right)+\sum_{v_{i} \in N_{G}(v) \backslash\{u\}} d_{G}\left(v_{i}\right)\right) \\
& -d_{G}(u) \sum_{u_{i} \in N_{G}(u) \backslash\{v\}} d_{G}\left(u_{i}\right)-d_{G}(v) \sum_{v_{i} \in N_{G}(v) \backslash\{u\}} d_{G}\left(v_{i}\right)-d_{G}(u) d_{G}(v) \\
= & \left(d_{G}(v)-1\right) \sum_{v_{i} \in N_{G}(u) \backslash\{v\}} d_{G}\left(u_{i}\right)+\left(d_{G}(u)-1\right) \sum_{v_{i} \in N_{G}(v) \backslash\{u\}} d_{G}\left(v_{i}\right) \\
& -\left(d_{G}(v)-1\right)\left(d_{G}(u)-1\right)
\end{aligned}
$$

and

$$
\begin{aligned}
M_{1}\left(G^{\prime}\right)-M_{1}(G) & =\left(d_{G}(u)+d_{G}(v)-1\right)^{2}+1-d_{G}(v)^{2}-d_{G}(u)^{2} \\
& =2\left(d_{G}(v)-1\right)\left(d_{G}(u)-1\right) .
\end{aligned}
$$

Also we have

$$
\sum_{v_{i} \in N_{G}(u) \backslash\{v\}} d_{G}\left(u_{i}\right) \geq d_{G}(u)-1 \text { and } \sum_{v_{i} \in N_{G}(v) \backslash\{u\}} d_{G}\left(v_{i}\right) \geq d_{G}(v)-1 .
$$

From (2.4) and (2.5), using (2.1) and (2.6) we obtain

$$
\begin{gathered}
\quad G R M_{\alpha}\left(G^{\prime}\right)-G R M_{\alpha}(G)=M_{2}\left(G^{\prime}\right)+\alpha M_{1}\left(G^{\prime}\right)-M_{2}(G)-\alpha M_{1}(G) \\
=\left(d_{G}(v)-1\right) \sum_{v_{i} \in N_{G}(u) \backslash\{v\}} d_{G}\left(u_{i}\right)+\left(d_{G}(u)-1\right) \sum_{v_{i} \in N_{G}(v) \backslash\{u\}} d_{G}\left(v_{i}\right) \\
+(2 \alpha-1)\left(d_{G}(v)-1\right)\left(d_{G}(u)-1\right) \\
=\left(d_{G}(v)-1\right)\left[\sum_{v_{i} \in N_{G}(u) \backslash\{v\}} d_{G}\left(u_{i}\right)+\left(\alpha-\frac{1}{2}\right)\left(d_{G}(u)-1\right)\right] \\
+\left(d_{G}(u)-1\right)\left[\sum_{v_{i} \in N_{G}(v) \backslash\{u\}} d_{G}\left(v_{i}\right)+\left(\alpha-\frac{1}{2}\right)\left(d_{G}(v)-1\right)\right] \\
\geq(2 \alpha+1)\left(d_{G}(v)-1\right)\left(d_{G}(u)-1\right) .
\end{gathered}
$$

(i) Since $\alpha>-1 / 2$ and $u v$ is a non-pendant cut edge in $G$, from (2.7), we get

$$
G R M_{\alpha}\left(G^{\prime}\right)>G R M_{\alpha}(G) .
$$

It contradicts the assumption that $G R M_{\alpha}(G)$ is maximum.

(ii) Since $\alpha=-1 / 2$ and $u v$ is a non-pendant cut edge in $G$, from (2.7), we get

$$
G R M_{\alpha}\left(G^{\prime}\right) \geq G R M_{\alpha}(G) \text {. }
$$

Suppose that equality holds in (2.9). Then from (2.6) and (2.7), we get $d_{G}\left(u_{i}\right)=1$ for $u_{i} \in N_{G}(u) \backslash\{v\}$ and $d_{G}\left(v_{i}\right)=1$ for $v_{i} \in N_{G}(v) \backslash\{u\}$. Hence $G$ is isomorphic to a double-star, but it contradicts the assumption. 
The number of cut edges of the considered graph $G^{\prime}$ in the proof of Proposition 2.6 is equal to the number of cut edges of $G$. i.e., If $G \in \mathcal{G}_{n}^{k}$, then also $G^{\prime} \in \mathcal{G}_{n}^{k}$. Hence we have the following corollary.

Corollary 2.7. Let $G$ be a graph in $\mathcal{G}_{n}^{k}$. Also let $G R M_{\alpha}(G)$ be maximum.

(i) If $\alpha>-1 / 2$ then all cut edges of $G$ are pendant.

(ii) If $\alpha=-1 / 2$ and $G$ is different from a double-star, then all cut edges of $G$ are pendant.

\section{Maximum $G R M_{\alpha}$ in $\mathcal{G}_{n}^{k}$ for $\alpha>-1 / 2$}

In this section, we give the sharp upper bound on $G R M_{\alpha}$ in $\mathcal{G}_{n}^{k}$ for $\alpha>-1 / 2$ and characterize the extremal graphs.

Proposition 3.1. Let $G$ be a graph in $\mathcal{G}_{n}^{k+}$ and $\alpha>-1 / 2$. If $G R M_{\alpha}(G)$ is maximum then $G$ is isomorphic to $K_{n}^{k}$.

Proof. If $k=n-1$ then $G$ is a tree of order $n$. Hence we get the required result by Corollary 2.4, because $K_{n}^{k} \cong S_{n}$. Let now $k<n-1$ and $G$ be a graph in $\mathcal{G}_{n}^{k+} \backslash\left\{K_{n}^{k}\right\}$ that is not isomorphic to $K_{n}^{k}$ such that $G_{R} M_{\alpha}(G)$ is maximum. Then we prove that

$$
\operatorname{GRM}_{\alpha}(G)<G R M_{\alpha}\left(K_{n}^{k}\right) .
$$

Let $u$ be a maximum degree vertex in $G$. Then $d_{G}(u)=n-1$, by Proposition 2.3. Hence all cut edges of $G$ are pendant. Let $l$ be the number of cut edges in $G$. Then $l \geq k$.

First we assume that $l>k$. Let $G^{\prime}$ be a graph obtained from $G$ by joining one pendant vertex to another non-pendant vertex of $G$. Then $G^{\prime} \in \mathcal{G}_{n}^{k+}$ and

$$
G R M_{\alpha}\left(G^{\prime}\right)>G R M_{\alpha}(G)
$$

by Proposition 2.5. It contradicts the fact that $G R M_{\alpha}(G)$ is maximum in $\mathcal{G}_{n}^{k+}$.

Next we assume that $l=k$. Then since $G$ is not isomorphic to $K_{n}^{k}$, there exist two non-adjacent vertices of degrees greater than one in the graph $G$. We join these two non-adjacent vertices and denote by $G^{\prime}$ the obtained graph. Then $G^{\prime} \in \mathcal{G}_{n}^{k+}$ and

$$
\operatorname{GRM}_{\alpha}\left(G^{\prime}\right)>G R M_{\alpha}(G)
$$

by Proposition 2.5. If $G^{\prime}$ is isomorphic to $K_{n}^{k}$ then we are done. Otherwise, a contradiction. This completes the proof.

Theorem 3.2. Let $G$ be a graph in $\mathcal{G}_{n}^{k}$ and $\alpha>-1 / 2$. Then

$$
\begin{gathered}
\operatorname{GRM}_{\alpha}(G) \leq \frac{1}{2}(n-k-1)(n-k-1+\alpha)[(n-k)(n-k-1+\alpha)+2 k] \\
+k(n-1+\alpha)(1+\alpha)
\end{gathered}
$$

with equality holding if and only if $G$ is isomorphic to $K_{n}^{k}$.

Proof. Since $K_{n}^{k} \in \mathcal{G}_{n}^{k}$ and $\mathcal{G}_{n}^{k} \subseteq \mathcal{G}_{n}^{k+}$, by Proposition 3.1, we have

$$
\operatorname{GRM}_{\alpha}(G)<G R M_{\alpha}\left(K_{n}^{k}\right)
$$

for all $G \in \mathcal{G}_{n}^{k}$ with $G \supsetneqq K_{n}^{k}$ and for $\alpha>-1 / 2$. By the definition of $G R M_{\alpha}$ we get

$$
\begin{aligned}
& \operatorname{GRM}_{\alpha}\left(K_{n}^{k}\right) \\
= & (n-1+\alpha)[(1+\alpha) k+(n-k-1+\alpha)(n-k-1)]+\left(\begin{array}{c}
n-k-1 \\
2
\end{array}\right)(n-k-1+\alpha)^{2} \\
= & (n-1+\alpha)(1+\alpha) k+(n-k-1)(n-k-1+\alpha)\left[n-1+\alpha+\frac{(n-k-2)(n-k-1+\alpha)}{2}\right] \\
= & (n-1+\alpha)(1+\alpha) k+\frac{1}{2}(n-k-1)(n-k-1+\alpha)[(n-k)(n-k-1+\alpha)+2 k] .
\end{aligned}
$$


From the above, we get the required result.

Corollary 3.3. $[5,10,11]$ Let $G$ be a graph in $\mathcal{G}_{n}^{k}$. Then

$$
M_{2}(G) \leq \frac{1}{2}(n-k-1)^{3}(n-k-2)+\left[(n-k-1)^{2}+k\right](n-1)
$$

with equality holding if and only if $G$ is isomorphic to $K_{n}^{k}$.

Proof. Taking $\alpha=0$ in Theorem 3.2, we get the required result.

\section{Maximum $G R M_{-1 / 2}$ in $\mathcal{G}_{n}^{k}$}

In this section, we give the sharp upper bound on $G R M_{-1 / 2}$ in $\mathcal{G}_{n}^{k}$ and characterize the extremal graphs.

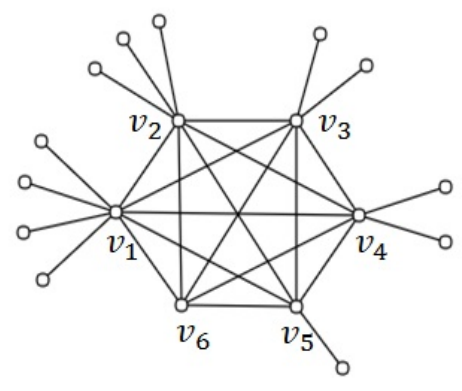

Fig. 1. The graph $G(4,3,2,2,1,0)$ in $\mathcal{G}(18,6) \subseteq \mathcal{G}_{18}^{12}$.

Let $N$ be a positive integer, $N \geq 1$. Denote by $K_{N}$, a complete graph of order $N$, and let $v_{1}, v_{2}, \ldots, v_{N}$ be its vertices. For $i=1,2, \ldots, N$, let $r_{i}$ be non-negative integers, labeled so that $r_{1} \geq r_{2} \geq \cdots \geq r_{N} \geq 0$. Construct the graph $G\left(r_{1}, r_{2}, \ldots, r_{N}\right)$ by attaching $r_{i}$ pendent vertices to the vertex $v_{i}$ of $K_{N}$. The graph $G\left(r_{1}, r_{2}, \ldots, r_{N}\right)$ has thus $n=N+\sum_{i=1}^{N} r_{i}$ vertices. For given values $n \geq N \geq 1$, the set of all graphs $G\left(r_{1}, r_{2}, \ldots, r_{N}\right)$ constructed in the above described manner is denoted by $\mathcal{G}(n, N)$ (see Fig. 1). If $N=1$ then $\mathcal{G}(n, 1)=\left\{S_{n}\right\}$ and if $N=2$ then $\mathcal{G}(n, 2)$ is the set of all double-stars of order $n$.

We now calculate the value on $G R M_{-1 / 2}(G)$ for the graphs $G$ in $\mathcal{G}(n, N)$.

Lemma 4.1. Let $G$ be a graph in $\mathcal{G}(n, n-k)$. Then

$$
G R M_{-1 / 2}(G)=\frac{1}{2}\left(n-\frac{3}{2}\right) k+\frac{1}{2}\left(n-k-\frac{3}{2}\right)(n-k-1)\left[(n-k)^{2}+\frac{7}{2} k-\frac{3}{2} n\right] .
$$

Proof. Since $G \in \mathcal{G}(n, n-k)$, there exist nonnegative integers $r_{1}, r_{2}, \ldots, r_{n-k}$, labeled so that $r_{1} \geq r_{2} \geq \cdots \geq r_{n-k} \geq 0$ with $r_{1}+r_{2}+\cdots+r_{n-k}=k$ and $G \cong G\left(r_{1}, r_{2}, \ldots, r_{n-k}\right)$. Let $v_{1}, v_{2}, \ldots, v_{n-k}$ be vertices of the graph $G\left(r_{1}, r_{2}, \ldots, r_{n-k}\right)$ whose degrees greater than one. Then $d_{G}\left(v_{i}\right)=r_{i}+n-k-1$ for $i=1,2, \ldots, n-k$,

$$
\sum_{i=1}^{n-k} r_{i}^{2}+2 \sum_{1 \leq i<j \leq n-k} r_{i} r_{j}=k^{2} \text { and } \sum_{1 \leq i<j \leq n-k}\left(r_{i}+r_{j}\right)=(n-k-1) k .
$$


Therefore, by using the above we get

$$
\begin{aligned}
& G R M_{-1 / 2}(G) \\
= & \sum_{i=1}^{n-k}\left(r_{i}+n-k-1-\frac{1}{2}\right)\left(1-\frac{1}{2}\right) r_{i} \\
& +\sum_{1 \leq i<j \leq n-k}\left(r_{i}+n-k-1-\frac{1}{2}\right)\left(r_{j}+n-k-1-\frac{1}{2}\right) \\
= & \frac{1}{2}\left(n-k-\frac{3}{2}\right) \sum_{i=1}^{n-k} r_{i}+\frac{1}{2} \sum_{i=1}^{n-k} r_{i}^{2}+\sum_{1 \leq i<j \leq n-k} r_{i} r_{j}+\left(n-k-\frac{3}{2}\right) \sum_{1 \leq i<j \leq n-k}\left(r_{i}+r_{j}\right) \\
= & \frac{1}{2}\left(n-k-\frac{3}{2}\right) k+\frac{1}{2} k^{2}+\left(n-k-\frac{3}{2}\right)^{2}\left(\begin{array}{c}
n-k \\
2
\end{array}\right) \\
= & \frac{1}{2}\left(n-\frac{3}{2}\right) k+\frac{1}{2}\left(n-k-\frac{3}{2}\right)(n-k-1)\left[(n-k)^{2}+\frac{7}{2} k-\frac{3}{2} n\right] .
\end{aligned}
$$


Fig. 2. All graphs $G$ in $\mathcal{G}(9,4) \subseteq \mathcal{G}_{9}^{5}$ with maximum value $G R M_{-1 / 2}(G)=93.75$.

If $G$ is a tree of order $n$ then $k=n-1$ and

$$
G R M_{-1 / 2}(G)=\frac{(2 n-3)(n-1)}{4}
$$

for $G \in\left\{S_{n}\right\} \cup \mathcal{G}(n, 2)$, by the above Lemma 4.1. From the definition of $\mathcal{G}(n, N)$, we have $\left\{S_{n}\right\} \cup \mathcal{G}(n, 2) \subseteq \mathcal{G}_{n}^{n-1}$ and $\mathcal{G}(n, N) \subseteq \mathcal{G}_{n}^{n-N}$ for $N \geq 3$. There is no connected graph of order $n$ with $n-2$ cut edges. Therefore, we further denote $\mathcal{G}(n, 1)=\left\{S_{n}\right\} \cup \mathcal{G}(n, 2)$.

Proposition 4.2. Let $G$ be a graph in $\mathcal{G}_{n}^{k}$. If $G R M_{-1 / 2}(G)$ is maximum, then $G \in$ $\mathcal{G}(n, n-k)$.

Proof. First, let $k=n-1$. If $G$ is different from a double-star then all cut edges of $G$ are pendant, by Corollary 2.7 (ii). Hence $G$ is isomorphic to star $S_{n}$ and $S_{n} \in \mathcal{G}(n, 1)$. If $G$ is isomorphic to a double-star, then $G \in \mathcal{G}(n, 1)$ and $G R M_{-1 / 2}(G)$ is also maximum in $\mathcal{G}_{n}^{n-1}$, because

$$
G R M_{-1 / 2}(G)=G R M_{-1 / 2}\left(S_{n}\right)
$$


from (4.1).

Let now $k<n-1$. Then $G$ is different from a tree. Hence by Corollary 2.7 (ii), all $k$ cut edges of $G$ are pendant. If $G \notin \mathcal{G}(n, n-k)$ then there exist two non-adjacent vertices of degrees greater than one in the graph $G$. We join these two non-adjacent vertices and denote by $G^{\prime}$ the obtained graph. Then $G^{\prime} \in \mathcal{G}_{n}^{k}$ and $G R M_{-1 / 2}\left(G^{\prime}\right)>G R M_{-1 / 2}(G)$ by Lemma 2.3. But it contradicts the fact that $G R M_{-1 / 2}(G)$ is maximum in $\mathcal{G}_{n}^{k}$.

Theorem 4.3. Let $G$ be a graph in $\mathcal{G}_{n}^{k}$. Then

$$
G R M_{-1 / 2}(G) \leq \frac{1}{2}\left(n-\frac{3}{2}\right) k+\frac{1}{2}\left(n-k-\frac{3}{2}\right)(n-k-1)\left[(n-k)^{2}+\frac{7}{2} k-\frac{3}{2} n\right]
$$

with equality holding if and only if $G \in \mathcal{G}(n, n-k)$.

Proof. If $G \in \mathcal{G}(n, n-k)$ then the equality holds in the above inequality for $G$, by Lemma 4.1. Otherwise the inequality is strict, by Proposition 4.2.

Example 4.4. By SageMath [23], we characterize all graphs in $\mathcal{G}_{9}^{5}$ that achieve the bound in Theorem 4.3 (see, Fig. 2).

Acknowledgment. This paper is dedicated to Prof. Ivan Gutman on the occasion of his 70th birthday. The authors are much grateful to the referees for their valuable comments on our paper, which have considerably improved the presentation of this paper. The first author was supported by Project No. 18105 from Mongolian National University of Education. The third author was supported by the National Research Foundation of the Korean government with grant No. 2017R1D1A1B03028642.

\section{References}

[1] B. Bollobás, P. Erdös, and Sarkar, A. Extremal graphs for weights, Discrete Math. 200, 5-19, 1999.

[2] B. Borovicanin and T.A. Lampert, On the maximum and minimum Zagreb indices of trees with a given number of vertices of maximum degree, MATCH Commun. Math. Comput. Chem. 74, 81-96, 2015.

[3] G. Caporossi and P. Hansen, Variable neighborhood search for extremal graphs. 5. Three ways to automate finding conjectures, Discrete Math. 276, 81-94, 2004.

[4] G. Caporossi, P. Hansen, and D. Vukičević, Comparing Zagreb indices of cyclic graphs, MATCH Commun. Math. Comput. Chem. 63, 441-451, 2010.

[5] S. Chen and W. Liu,Extremal Zagreb indices of graphs with a given number of cut edges, Graphs and Combinatorics 30, 109-118, 2014.

[6] K.C. Das, Maximizing the sum of the squares of the degrees of a graph, Discrete Math. 285, 57-66, 2004.

[7] K.C. Das and I. Gutman, Some properties of the second Zagreb index, MATCH Commun. Math. Comput. Chem. 52, 103-112, 2004.

[8] K.C. Das, I. Gutman, and B. Horoldagva, Comparison between Zagreb indices and Zagreb coindices of trees, MATCH Commun. Math. Comput. Chem. 68, 189-198, 2012.

[9] C. Elphick and T. Reti, On the relations between the Zagreb indices, clique numbers and walks in graphs, MATCH Commun. Math. Comput. Chem. 74, 19-34, 2015.

[10] Y. Feng, X. Hu, and S. Li, On the extremal Zagreb indices of graphs with cut edges, Acta Appl. Math. 110, 667-684, 2010. 
[11] Y. Feng, X. Hu, and S. Li, Erratum to: On the extremal Zagreb indices of graphs with cut edges, Acta Appl. Math. 110, 685, 2010.

[12] B. Furtula, I. Gutman, and S. Ediz, On difference of Zagreb indices, Discrete Appl. Math. 178, 83-88, 2014.

[13] I. Gutman, B. Furtula, and C. Elphick, Three new/old vertex degree-based topological indices, MATCH Commun. Math. Comput. Chem. 72, 617-682, 2014.

[14] I. Gutman, B. Ruščić, N. Trinajstić, and C.F. Wilcox, Graph theory and molecular orbitals, XII. Acyclic polyenes, J. Chem. Phys. 62, 3399-3405, 1975.

[15] I. Gutman and N. Trinajstić, Graph theory and molecular orbitals. Total $\pi$-electron energy of alternant hydrocarbons, Chem. Phys. Lett. 17 535-538, 1971.

[16] P. Hansen and D. Vukičević, Comparing the Zagreb indices, Croat. Chem. Acta 80, 165-168, 2007.

[17] B. Horoldagva, L. Buyantogtokh, and S. Dorjsembe, Difference of Zagreb indices and reduced second Zagreb index of cyclic graphs with cut edges, MATCH Commun. Math. Comput. Chem. 78, 337-349, 2017.

[18] B. Horoldagva and K.C. Das, On comparing Zagreb indices of graphs, Hacettepe J. of Math. and Stat., 41, 223-230, 2012.

[19] B. Horoldagva, K.C. Das, and T. Selenge, Complete characterization of graphs for direct comparing Zagreb indices, Discrete Appl. Math. 215, 146-154, 2016.

[20] B. Horoldagva and S.-G. Lee, Comparing Zagreb indices for connected graphs, Discrete Appl. Math. 158, 1073-1078, 2010.

[21] U.N. Peled, R. Petreschi, and A. Sterbini, $(n, e)$-graphs with maximum sum of squares of degrees, J. Graph Theory 31, 283-295, 1999.

[22] T. Selenge and B. Horoldagva, Maximum Zagreb indices in the class of $k$-apex trees, Korean J. Math. 23, 401-408, 2015.

[23] http://math3.skku.ac.kr

[24] D. Stevanović and M. Milanič, Improved inequality between Zagreb indices of trees, MATCH Commun. Math. Comput. Chem. 68, 147-156, 2012.

[25] L. Sun and T. Chen, Comparing the Zagreb indices for graphs with small difference between the maximum and minimum degrees, Discrete. Appl. Math. 157, 1650-1654, 2009.

[26] D. Vukičević and A. Graovac, Comparing Zagreb $M_{1}$ and $M_{2}$ indices for acyclic molecules, MATCH Commun. Math. Comput. Chem. 57, 587-590, 2007.

[27] H. Wang and S. Yuan, On the sum of squares of degrees and products of adjacent degrees, Discrete Math. 339, 1212-1220, 2016. 